\title{
The Milky Way rotation curve revisited
}

\author{
D. Russeil ${ }^{1}$, A. Zavagno ${ }^{1}$, P. Mège ${ }^{1}$, Y. Poulin ${ }^{1}$, S. Molinari ${ }^{2}$, and L. Cambresy ${ }^{3}$ \\ 1 Aix Marseille Univ., CNRS, LAM, Laboratoire d'Astrophysique de Marseille, Marseille, France \\ e-mail: delphine.russeil@lam.fr \\ 2 Istituto Nazionale di Astrofisica - IAPS, via Fosso del Cavaliere 100, 00133 Roma, Italy \\ 3 Observatoire Astronomique de Strasbourg, Université de Strasbourg, CNRS, UMR 7550, 11 rue de l'Université, 67000 Strasbourg, \\ France
}

Received 1 February 2017 / Accepted 11 April 2017

\begin{abstract}
The Herschel survey of the Galactic Plane (Hi-GAL) is a continuum Galactic plane survey in five wavebands at 70, 160, 250, 350 and $500 \mu \mathrm{m}$. From such images, about 150000 sources have been extracted for which the distance determination is a challenge. In this context the velocity of these sources has been determined thanks to a large number of molecular data cubes. But to convert the velocity to kinematic distance, one needs to adopt a rotation curve for our Galaxy. For three different samples of tracers, we test different analytical forms. We find that the power-law expression, $\theta(R) / \theta_{0}=1.022\left(R / R_{0}\right)^{0.0803}$ with $R_{0}, \theta_{0}=8.34 \mathrm{kpc}, 240 \mathrm{~km} \mathrm{~s}{ }^{-1}$ is a good and easily manipulated expression for the distance determination process.
\end{abstract}

Key words. Galaxy: disk - Galaxy: kinematics and dynamics

\section{Introduction}

In this paper we propose to update the rotation curve of our Galaxy. This is done to determine the distance of the sources extracted from the Hi-GAL survey (e.g. Molinari et al. 2016). This open time key-project of the Herschel Space Observatory (Pilbratt et al. 2010) is a 5-band photometric imaging survey (with the SPIRE and PACS photometric cameras) at 70, 160, 250,350 , and $500 \mu \mathrm{m}$ of a $|b| \leq 1^{\circ}$ wide strip of the Milky Way Galactic plane.

In the context of the Vialactea Project ${ }^{1}$ a large number of molecular data cubes, covering the full galactic plane, have been compiled (Molinaro et al. 2016) from which velocity for the HiGAL sources are extracted (Mège et al., in prep.). To convert this velocity into kinematic distance we need to adopt a rotation curve for our Galaxy.

Up to now, two main rotation curves have generally been used: the Clemens (1985) and the Brand \& Blitz (1993) curves. To determine the global rotation curve it is important to have access to data from all the galactic quadrants and to combine the three usual methods to link the velocity to the distance. Usually $\mathrm{CO}$ and/or H I observations of the interstellar medium are used to establish the inner (inner the Solar circle) rotation curve (assuming circular rotation) thanks to the tangent-point method (which used the terminal line-of-sight velocities). Such an approach is complemented adding the $\mathrm{H}$ II regions (or star-forming complexes) to trace the external part (outside the Solar circle) of the rotation curve because for H II regions one can independently measure the velocity of the gas and the distance of the exciting stars. Recently, a third method based on the maser parallaxes produced a new set of data. Indeed, maser(s) observed in star-forming regions are used to determine their parallactic distance and are very useful to determine the distance of embedded

\footnotetext{
1 http://vialactea-sg.oact.inaf.it:8080/web/guest/ home
}

star-formation regions for which the classical exciting OB stars are not observable. Recently, Reid et al. $(2009,2014)$ used maser parallax distances of star-forming regions to trace the rotation curve. However, these new results are determined from a "small" number (100) of high-mass star-forming regions and from the northern part of the galactic plane $\left(l \sim 0^{\circ}\right.$ to $\left.240^{\circ}\right)$. Because of the long-known asymmetry (Kerr 1964; Georgelin \& Georgelin 1976; Blitz \& Spergel 1991; Levine et al. 2008) between the southern and the northern rotation curves, to determine the distance of any source in our Galaxy it is better to use a rotation curve established from the full range of longitudes (Gómez 2006). It is in this context that we revisit the Milky Way rotation curve.

\section{The sample}

The best method to determine the rotation curve of our galaxy is to independently measure the velocity and the distance (exciting star or maser parallax distances) of the objects. However, this method limits probing the local Galaxy only (the stellar distance can be evaluated only up to about $6 \mathrm{kpc}$ due to extinction, and maser parallax in star-forming regions requires long time baseline). To probe the inner rotation of our Galaxy on a larger scale the $\mathrm{HI} / \mathrm{CO}$ tangent method is usually used. If this method can have uncertainties due to local motions, Chemin et al. (2015) show that it is adequate for galactocentric distances larger than $4.5 \mathrm{kpc}$.

We propose here to update the rotation curve of our Galaxy by combining different samples:

1. Sample 1: the H II regions/complexes-stellar distance cataloged by Brand \& Blitz (1993). Their H II regions catalog has been downloaded from Vizier ${ }^{2}$ and provides, for every

\footnotetext{
2 http://vizier.u-strasbg.fr/viz-bin/VizieR?-source=
} $\mathrm{J} / \mathrm{A}+\mathrm{A} / 275 / 67$ 
object, the $l, b$ coordinates, the $V_{\mathrm{LSR}}$, and the stellar distance. This catalog provides a sample of 152 objects (select to have $\left.|b|<3^{\circ}\right)$ distributed in the four galactic quadrants.

2. Sample 2: the maser parallax distance catalogs of starforming regions from Reid et al. (2014) and Honma et al. (2012). We retrieved the catalogs from Reid et al. (2014) and Honma et al. (2012). These catalogs give, for each object, the $1, \mathrm{~b}$ coordinates, the $V_{\mathrm{LSR}}$ (CO velocity) of the associated molecular cloud, and the parallax $\pi$ (which is then converted into distance as $\left.d_{\pi}=1 / \pi\right)$. Both catalogs give a final sample of 101 objects (selected to have distance larger than $1 \mathrm{kpc}$ ) located mainly in quadrants 1 and 2 of our Galaxy.

3. Sample 3: the HI tangent+CO-H II regions catalog from Sofue et al. (2009). They compiled H I tangent point data from Burton \& Gordon (1978), Clemens (1985) and Fich et al. (1989), H I-disk thickness method from Honma \& Sofue (1997a,b), CO and H II regions from Fich et al. (1989) and Blitz et al. (1982). This data provides, for every object, the rotation velocity and the galactocentric distance (calculated with $R_{0}, \theta_{0}=8 \mathrm{kpc}, 200 \mathrm{~km} \mathrm{~s}^{-1}$ ). Selecting data with velocity uncertainty less than $50 \mathrm{~km} \mathrm{~s}^{-1}$ and rotation velocity between 150 and $350 \mathrm{~km} \mathrm{~s}^{-1}$ gives a sample of 408 measurements.

4. Sample 4: the Hi tangent measurements from McClureGriffiths \& Dickey (2007) and McClure-Griffiths \& Dickey (2016). They are based on the Southern Galactic Plane survey (McClure-Griffiths et al. 2005) and the VLA galactic plane survey (Stil et al. 2006). The northern and southern survey cover $18^{\circ} \leq l \leq 67^{\circ}$ (with latitude varying from $|b|<1.3^{\circ}$ to $\left.|b|<2.3^{\circ}\right)$, and $253^{\circ} \leq l \leq 358^{\circ}\left(|b| \leq 1.5^{\circ}\right)$, respectively. Selecting only the data with galactocentric distance larger than $4 \mathrm{kpc}$, gives a sample of 1243 measurements. In their data tables, no velocity uncertainty is given, so we adopt a $10 \mathrm{~km} \mathrm{~s}^{-1}$ uncertainty.

\section{The adopted local standard of rest and solar motion parameters}

Up to now, to establish the $V_{\mathrm{LSR}}$, and to determine the distance, assumptions have been made concerning the Solar parameters: first the Solar motions $\left(U_{\odot}, V_{\odot}, W_{\odot}\right)$ to the local standard of rest (LSR) and the LSR parameters, which are the distance of the Sun to the galactic center $\left(R_{0}\right)$ and the rotation velocity $\left(\theta_{0}\right)$. The IAU standard values for these quantities are $R_{0}=8.5 \mathrm{kpc}$, $\theta_{0}=220 \mathrm{~km} \mathrm{~s}^{-1}, U_{\odot}=10.27 \mathrm{~km} \mathrm{~s}^{-1}, V_{\odot}=15.32 \mathrm{~km} \mathrm{~s}^{-1}$, and $W_{\odot}=7.74 \mathrm{~km} \mathrm{~s}^{-1}$. One can recall that $U_{\odot}, V_{\odot}, W_{\odot}$ are exclusively used to calculate the $V_{\mathrm{LSR}}$ from the measured heliocentric radial velocity while $R_{0}$ and $\theta_{0}$ are used in the kinematic distance determination. From maser parallaxes, new $R_{0}, \theta_{0}$ values were also determined as being $8.05 \pm 0.45 \mathrm{kpc}, 238 \pm 14 \mathrm{~km} \mathrm{~s}^{-1}$ and $8.34 \pm 0.16 \mathrm{kpc}, 240 \pm 8 \mathrm{~km} \mathrm{~s}^{-1}$ by Honma et al. (2012) and Reid et al. (2014), respectively. Such a low $R_{0}$ value (between 7.7 and $8.27 \mathrm{kpc}$ ) is also found from independent measurements (Meyer et al. 2012; Gillessen et al. 2013; Chatzopoulos et al. 2015) as recently underlined by Boehle et al. (2016) who find $R_{0}=7.86 \mathrm{kpc}$. In parallel, $\omega_{0}$, determined from different approaches (Feast \& Whitelock 1997; Reid \& Brunthaler 2004; Reid et al. 2014; Bobylev 2017), is between 27.19 and $29.45 \mathrm{~km} \mathrm{~s}^{-1} \mathrm{kpc}^{-1}$ which implies a $\theta_{0}$ value larger than the IAU one.

Reid et al. $(2009,2014)$ suggested also from the 3D motion measured for masers that $U_{\odot}, V_{\odot}$, and $W_{\odot}$ must be updated and that the particular motion of the sources must be taken into account. Unfortunately, for Hi-GAL sources, we will have no information about their own $U, V, W$. We therefore assume them to be null. Several other authors suggested alternative values to the standard ones for $U_{\odot}, V_{\odot}, W_{\odot}$, and $R_{0}, \theta_{0}$ (we refer to Hou \& Han 2014, for a revue on this). If two main sets of " $R_{0}$, $\theta_{0}$ " emerge (the IAU standard one and the $R_{0}, \theta_{0}=8.34 \mathrm{kpc}$, $240 \mathrm{~km} \mathrm{~s}^{-1}$ ) for $U_{\odot}, V_{\odot}$, and $W_{\odot}$, no general agreement, especially for $V_{\odot}$, is brought out. However, because in the frame of the Hi-GAL survey we use source velocity extracted from different $l, b, V_{\mathrm{LSR}}$ data cubes (Molinaro et al. 2016) we can expect that, by default, their $V_{\mathrm{LSR}}$ is calculated with the $U_{\odot}, V_{\odot}, W_{\odot}$ IAU standard.

\section{The updated version of the Galactic rotation curve}

To produce an updated version of the rotation curve, we used the data listed above and fitted different analytical expressions. From the data, we define three distinct sub-samples: one combining samples 1, 2 and 3 ("Sub 123"), one combining samples 1 and 2 ("Sub 12") and one combining samples 1, 2 and 4 ("Sub 124"). To avoid redundancy, in "Sub 123" we only add the $74 \mathrm{H}$ II regions of sample 1 not in common with the ones already used in sample 3. In "Sub 12" the H II regions (all the regions from sample 1) and masers are put together to probe the rotation curve as traced by a similar method (velocity independent of the distance calculation). To avoid redundancy, we do not combine samples 3 and 4. However, as mentioned by McClure-Griffiths \& Dickey (2016) comparing CO and H I data, we checked the good agreement between them.

Figure A.1 shows the different sub-samples and the typical error bars. By default, the tangent method always gives very small error bars and smaller scattering with respect to the H II regions and masers. In parallel, the galactocentric range around $8.5 \mathrm{kpc}$ is naturally well populated by $\mathrm{H}$ II regions because at larger distance from the Sun the extinction no longer allows stellar distance determination. In the $R \sim 6 \mathrm{kpc}$ to $8 \mathrm{kpc}$ the tangent method data points and H II regions agree, while below $6 \mathrm{kpc}$, the masers show a clear offset from them.

In the literature, several expressions for the rotation curve are used:

- A polynomial form: $\theta(R) / \theta_{0}=a_{1}+a_{2} r+a_{3} r^{2}$ with $r=$ $\left(\left(R / R_{0}\right)-1\right)$ used by Reid et al. (2014).

- Power law forms: Honma et al. (2012) and Brand \& Blitz (1993) used the following power law forms $\theta(R) / \theta_{0}=a_{1}$ $\left(\mathrm{R} / R_{0}\right)^{a_{2}}$ and $\theta(R) / \theta_{0}=a_{1}\left(\mathrm{R} / R_{0}\right)^{a_{2}}+a_{3}$, respectively.

- A universal form: Persic et al. (1996) suggest a more universal form $\left.\left(\theta(R) / \theta_{0}=a_{1}\left[1+a_{2}\left(\left(R / a_{3}\right)-1\right)\right]\right)\right)$ based on a sample of extragalactic rotation curves.

- The Polyex model: Giovanelli \& Haynes (2002) used, to fit rotation curves for 2246 galaxies, another universal analytical expression (known as the "polyex" model) with the form $\theta(R) / \theta_{0}=\left(1-e^{-R / a_{1}}\right) \times\left(1+\left(a_{2} R / a_{1}\right)\right)$.

Before performing the fit we scale the data to the chosen $R_{0}$, $\theta_{0}$ set, following, for example, Xin \& Zheng (2013). To compare with the old and new results, we performed the fit on the three sub-samples with both $R_{0}=8.5 \mathrm{kpc}, \theta_{0}=220 \mathrm{~km} \mathrm{~s}^{-1}$ and $R_{0}=8.34 \mathrm{kpc}, \theta_{0}=240 \mathrm{~km} \mathrm{~s}^{-1}$ sets. In practice, following Fich et al. (1989), the rotation curves are fitted in $\omega$ versus $R$, because they are observationally independent quantities. We requested also that the cataloged objects have $|b|<3^{\circ}$ (objects with larger latitude are probably close objects for which the systemic velocity can be distorted by local motions) and 
$R>4 \mathrm{kpc}$ because closer to the Galactic center the contribution of the bulge and the bar to the kinematics can become important (e.g. Chemin et al. 2015; Reid et al. 2014). The fits are done minimizing the normalised weighted $\chi^{2}$ expression (where the weight is the inverse of the squared uncertainty) using the "Minuit" subroutine (Nelder \& Mead 1965). The different fitted solutions are shown in Figs. A.2 and A.3 for the both adopted $R_{0}, \theta_{0}$ sets, while the results are listed in Table A.1. Whatever the model, the value of the standard normalised $\chi^{2}$ we found is small (between 0.09 and 0.45 ) while it is expected around unity. A value less than one does not necessary indicate a better fit but underlines uncertainty in the determination of the variance (Bevington \& Robinson 2003). Despite the fact that it gives indication about the data dispersion around the fited curve, Fich et al. (1989) show that the $\chi^{2}$ numerical value cannot be easily used to sort the goodness of fit analysis. We then also used the evaluated parameter uncertainties ${ }^{3}$ to compare the fits because they are related to the width of the minimized expression minimum.

Looking at the figures we note the all the fitted curves give similar results except the polynomial form which, for several configurations, departs from the others after $R=8 \mathrm{kpc}$. From "Sub12", we note also that all the models fit well the data in the range 6 to $10 \mathrm{kpc}$, while below $6 \mathrm{kpc}$ they are not able to fit the regions offset from the tangent data. This underlines the fact that adding the tangent data will not impact too much the fitted curve in such inner parts and that fixing $R_{0}, \theta_{0}$ strongly forces the fit in this distance range.

\section{Discussion}

To compare our results with previous studies we have to keep in mind that we fit with fixed $R_{0}$ and $\theta_{0}$, as did McClure-Griffiths \& Dickey (2016) and Levine et al. (2008), for example, while Reid et al. (2014) and Honma et al. (2012), for example, have them as free parameters. In addition, Reid \& Dame (2016) found that a slightly curved rotation curve with $\theta_{0}=240 \mathrm{~km} \mathrm{~s}^{-1}$ can mimic a flat rotation curve with $\theta_{0}=220 \mathrm{~km} \mathrm{~s}^{-1}$, convincing us to fit with fixed $R_{0}$ and $\theta_{0}$. However fixing $R_{0}$ and $\theta_{0}$ implies some expected relation between the fitted parameters. For example, $a 1 \sim 1$ is expected for Polynomial and Power-law models while $a 3$ and $a 1$ close to $R_{0}$ is expected for the Universal and Polyex models, respectively. Any large departure from these expected values underlines a least good fit, however, for most of the fitted parameters and the expected values are in agreement.

A possible approach followed by several authors to study the rotation curve is to simply fit a linear expression of the form $\theta(R) / \theta_{0}=a_{1}+a_{2}\left(R / R_{0}\right)$. Some authors fit only the inner part of the rotation curve ( 3 to $8 \mathrm{kpc}$ ), because this is traced from the tangent velocity method. This is the case for Fich et al. (1989) for quadrant I, Levine et al. (2008) for quadrants I and IV and McClure-Griffiths \& Dickey (2016) for quadrant I, who find $\left(a_{1}\right.$, $\left.a_{2}\right)=(0.887,0.186),(0.855,0.024),(0.829,0.026),(0.82,0.026)$, respectively. Others fit a linear rotation curve to data within 4 and $16 \mathrm{kpc}$ (Reid et al. 2014) or even within 8 and $11 \mathrm{kpc}$ (Huang et al. 2016) finding $\left(a_{1}, a_{2}\right)=\left(1.007,-8.3 \times 10^{-3}\right)$ and $(1.23,-0.023)$, respectively. We note that focussing on the inner rotation curve gives a positive slope while fitting up to a larger radius changes the slope to negative (but close to zero value).

We first test the polynomial model. In this model $a_{1} \times \theta_{0}$ gives the overall amplitude of the rotation curve, and $a_{2}$ and $a_{3}$

\footnotetext{
3 See the Minuit reference manual at https://root.cern.ch/ sites/d35c7d8c. web.cern.ch/files/minuit.pdf
}

describe the position of the curve extremum (with respect to $R_{0}$ ) and the curve inflection, respectively. For a decreasing curve, $a_{3}$ must be negative while the smaller $\left|a_{3}\right|$ is, the flatter the curve. Reid et al. (2014), fitting such a form to masers, found $a_{1}, a_{2}$, $a_{3}=1,0.002,-0.06$ (following our parameter definitions). Focussing on the two curves of "Sub12", we find a similar $a_{1}$ but a systematically larger $\left|a_{3}\right|$ suggesting our curves are more decreasing. However, we can note that the polynomial form consistently gives the worst $\chi^{2}$ with respect to the other forms as can also be seen in Figs. A.2 and A.3.

In the "polyex" expression, Giovanelli \& Haynes (2002) describe $a_{1}$ as the scale length for the inner steep rise (the radial distance at which $\theta$ is 0.63 of the asymptotic velocity for a flat rotation curve) while $a_{2}$ sets the slope of the rotation curve's outer part. From approximately 2200 low-redsift galaxies, Catinella et al. (2006) show that $0.002<a_{2}<$ 0.087 . However, for galaxies with maximum velocity around $220-240 \mathrm{~km} \mathrm{~s}^{-1}, a_{2}$ is expected to be between approximately 0.003 and 0.006. Similar values are found for "Sub 123" and "Sub 124" while for "Sub 12" it is found negative but still close to zero. In Catinella et al. (2006), $a_{1}$ is in units of exponential disk scale length $\left(R_{\mathrm{d}}\right)$ and for galaxies with maximum velocity around $220-240 \mathrm{~km} \mathrm{~s}^{-1}$, it is estimated around 0.45 . With a disk scale length for our Galaxy between $2.15 \mathrm{kpc}$ (Bovy \& Rix 2013) and $3.19 \mathrm{kpc}$ (Sofue 2012) we expect $a_{1}$ to be between 1.07 and $1.43 \mathrm{kpc}$. But whatever our sample, $a_{1}$ is found around $1 \mathrm{kpc}$ and even smaller for "Sub 12" (with the IAU $R_{0}, \theta_{0}$ ).

In the Universal law expression, $a_{1} \times \theta_{0}$ is the maximum velocity, $a_{3}$ is the radius where this maximum velocity (also noted $R_{\max }$ ) is reached and $a_{2}$ is the velocity variation between $a_{3}$ and $R_{\text {opt }}$. As expected, $a_{1}$ is close to 1 whatever the sample. Persic et al. (1996) find $-0.1 \leq a_{2} \leq 0.6$. We find such values for all our fitting configurations. From Persic et al. (1996), usually $a_{3} \sim 2.2 \times R_{\mathrm{d}}$, which suggest $a_{3}$ between $4.7 \mathrm{kpc}$ and $7 \mathrm{kpc}$ for our Galaxy. For $R_{0}=8.5 \mathrm{kpc}, a_{3}$ is found very close to $R_{0}$ while for $R_{0}=8.34 \mathrm{kpc}$ a departure is noted reaching $40 \%$ for "Sub 123". Reid et al. (2014) also fit such a Universal law to masers and find $a_{1}, a_{2}, a_{3}=1,0.003,12.13 \mathrm{kpc}$ (following our parameter definitions and with their $R_{0}, \theta_{0}=8.31 \mathrm{kpc}$ and $241 \mathrm{~km} \mathrm{~s}^{-1}$ ) which suggests a flatter rotation curve.

In the power law forms, $a_{2}$, the exponent, describes how quickly the curve decreases/increases while $a_{3}$ is the deviation term, which represents a simple way for observations to deviate from the power-law function. We note that $a_{1}+a_{3} \sim 1$, as expected for the Brand \& Blitz (1993) form, is well recovered for IAU $R_{0}, \theta_{0}$ while it is slightly smaller for $R_{0}, \theta_{0}=$ $8.34 \mathrm{kpc}$ and $240 \mathrm{~km} \mathrm{~s}^{-1}$. In addition to their H II regions catalog, Brand \& Blitz (1993) added H I tangent velocities to compute a rotation curve (with $R_{0}, \theta_{0}=8.5 \mathrm{kpc}, 220 \mathrm{~km} \mathrm{~s}^{-1}$ ) with the power law form and found $a_{1}, a_{2}, a_{3}=1.00767,0.0394,0.00712$ similar to our results obtained for "Sub 123" and "Sub 124".

\section{Conclusion}

Testing different analytical forms to different samples we find that all the forms, except the polynomial one, give satisfactory fitting results. The two power-law forms are often superimposed. The models used in extra-galactic studies (universal and polyex forms) are also tested. However they implement a radius scaling parameter which is difficult to relate to $R_{0}$ as is the case for the other form. Using a stellar/maser distance sample gives more departures, even if the fits are good, between the different forms; in particular in the outer part of the rotation curve. In addition, 
in the inner part, the fitted curve is not able to pass through the data points but passes at the expected location of the tangent point as plotted by "Sub 123" and "Sub 124". The power-law appears then as the simplest and easiest form describing the rotation curve, and because the two power forms are often superimposed, we favour the simplest form (with no $a_{3}$ ). In the frame of the kinematic distance determination of the Hi-GAL sources, we then adopt the power law form $\theta(R) / \theta_{0}=1.022\left(R / R_{0}\right)^{0.0803}$ with $R_{0}, \theta_{0}=8.34 \mathrm{kpc}, 240 \mathrm{~km} \mathrm{~s}^{-1}$.

To improve the Galactic rotation curve it appears important to better sample its outer part $(R>10 \mathrm{kpc})$. In this framework, we expect that the incoming ESA-Gaia database and maser parallactic distances will provide such information. Indeed, the ESA-Gaia database should provide better distance and rotation velocity determination (and velocity field) for the OB stars exciting the H II regions and identify and quantify the circular velocity departures of such regions (in the detection limits). It will also allow one to trace the galactic rotation curve (and the velocity field) given by the stellar background potential and to compare it to the observed one, as it is expected (Gómez 2006) that the observed rotation curve is systematically above the true one. Maser parallax distances appear also as a very accurate and promising tool (and complementary to Gaia) for directly determining the distance of the star-forming regions in which Hi-GAL sources are located.

Acknowledgements. This work is part of the VIALACTEA Project, a Collaborative Project under Framework Programme 7 of the European Union, funded under Contract \# 607380 that is hereby acknowledged.

\section{References}

Bevington, P. R., \& Robinson, D. K. 2003, Data reduction and error analysis for the physical sciences (McGraw-Hill)

Blitz, L., \& Spergel, D. N. 1991, ApJ, 370, 205

Blitz, L., Fich, M., \& Stark, A. A. 1982, ApJS, 49, 183
Bobylev, V. V. 2017, Astron. Lett., in press, ArXiv e-prints [arXiv: 1611.01766]

Boehle, A., Ghez, A. M., Schödel, R., et al. 2016, ApJ, 830, 17 Bovy, J., \& Rix, H.-W. 2013, ApJ, 779, 115

Brand, J., \& Blitz, L. 1993, A\&A, 275, 67

Burton, W. B., \& Gordon, M. A. 1978, A\&A, 63, 7

Catinella, B., Giovanelli, R., \& Haynes, M. P. 2006, ApJ, 640, 751

Chatzopoulos, S., Fritz, T. K., Gerhard, O., et al. 2015, MNRAS, 447, 948

Chemin, L., Renaud, F., \& Soubiran, C. 2015, A\&A, 578, A14

Clemens, D. P. 1985, ApJ, 295, 422

Feast, M., \& Whitelock, P. 1997, MNRAS, 291, 683

Fich, M., Blitz, L., \& Stark, A. A. 1989, ApJ, 342, 272

Georgelin, Y. M., \& Georgelin, Y. P. 1976, A\&A, 49, 57

Gillessen, S., Eisenhauer, F., Fritz, T. K., et al. 2013, in Advancing the Physics of Cosmic Distances, ed. R. de Grijs, IAU Symp., 289, 29

Giovanelli, R., \& Haynes, M. P. 2002, ApJ, 571, L107

Gómez, G. C. 2006, AJ, 132, 2376

Honma, M., \& Sofue, Y. 1997a, PASJ, 49, 539

Honma, M., \& Sofue, Y. 1997b, PASJ, 49, 453

Honma, M., Nagayama, T., Ando, K., et al. 2012, PASJ, 64, 136

Hou, L. G., \& Han, J. L. 2014, A\&A, 569, A125

Huang, Y., Liu, X.-W., Yuan, H.-B., et al. 2016, MNRAS, 463, 2623

Kerr, F. J. 1964, in The Galaxy and the Magellanic Clouds, ed. F. J. Kerr, IAU Symp., 20, 81

Levine, E. S., Heiles, C., \& Blitz, L. 2008, ApJ, 679, 1288

McClure-Griffiths, N. M., \& Dickey, J. M. 2007, ApJ, 671, 427

McClure-Griffiths, N. M., \& Dickey, J. M. 2016, ApJ, 831, 124

McClure-Griffiths, N. M., Dickey, J. M., Gaensler, B. M., et al. 2005, ApJS, 158, 178

Meyer, L., Ghez, A. M., Schödel, R., et al. 2012, Science, 338, 84

Molinari, S., Schisano, E., Elia, D., et al. 2016, A\&A, 591, A149

Molinaro, M., Butora, R., Bandieramonte, M., et al. 2016, Proc. SPIE, 9913, $99130 \mathrm{H}$

Nelder, J., \& Mead, R. 1965, Comput. J., 7, 308

Persic, M., Salucci, P., \& Stel, F. 1996, MNRAS, 281, 27

Pilbratt, G. L., Riedinger, J. R., Passvogel, T., et al. 2010, A\&A, 518, L1

Reid, M. J., \& Brunthaler, A. 2004, ApJ, 616, 872

Reid, M. J., \& Dame, T. M. 2016, ApJ, 832, 159

Reid, M. J., Menten, K. M., Zheng, X. W., et al. 2009, ApJ, 700, 137

Reid, M. J., Menten, K. M., Brunthaler, A., et al. 2014, ApJ, 783, 130

Sofue, Y. 2012, PASJ, 64, 75

Sofue, Y., Honma, M., \& Omodaka, T. 2009, PASJ, 61, 227

Stil, J. M., Taylor, A. R., Dickey, J. M., et al. 2006, AJ, 132, 1158

Xin, X.-S., \& Zheng, X.-W. 2013, Res. Astron. Astrophys., 13, 849 
D. Russeil et al.: The Milky Way rotation curve revisited

Appendix A: Figures and table
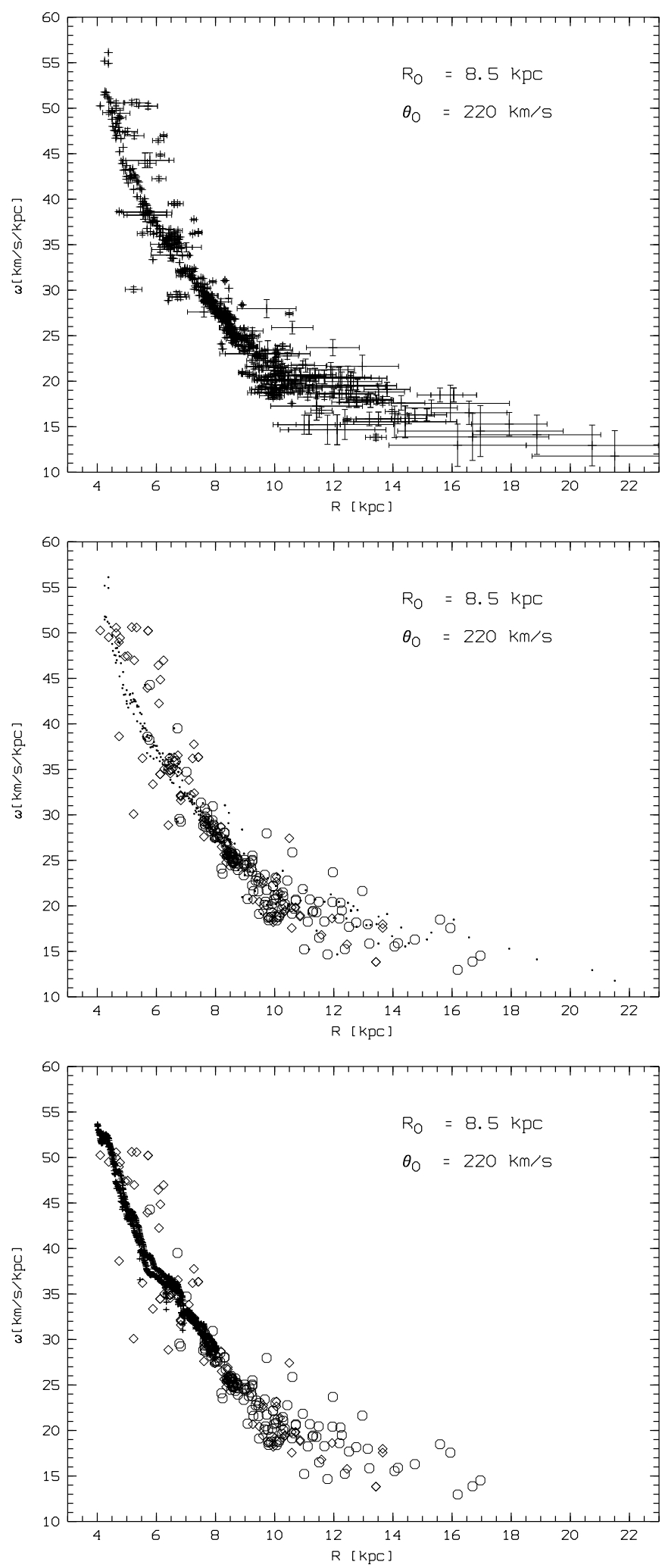

Fig. A.1. Data sample used for the rotation curve fitting. The upper panel shows the error bars. The middle panel shows sources from sample 1 (circles), sample 2 (diamonds), and sample 3 (dots), respectively. The lower panel shows sample 4 (dots) instead of sample 3 while the other symbols are similar as in middle panel.
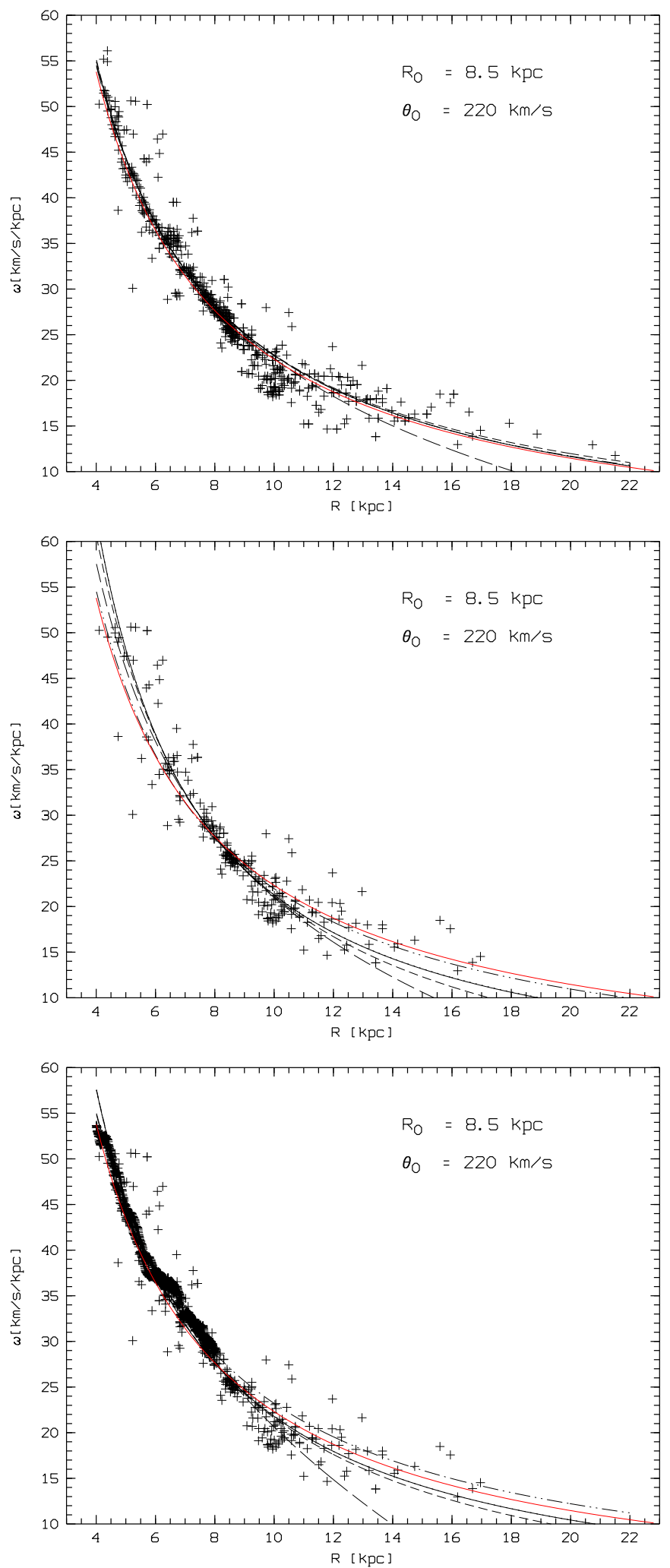

Fig. A.2. Fitted rotation curves with $R_{0}, \theta_{0}$ set to $8.5 \mathrm{kpc}$ and $220 \mathrm{~km} \mathrm{~s}^{-1}$, respectively. The fitted Brand \& Blitz (1993), power law, polynomial, Universal and polyex forms are displayed as solid, dotted, long dash, short dash, and dash-dot lines, respectively. The Brand \& Blitz (1993) rotation curve is superimposed (red line). 

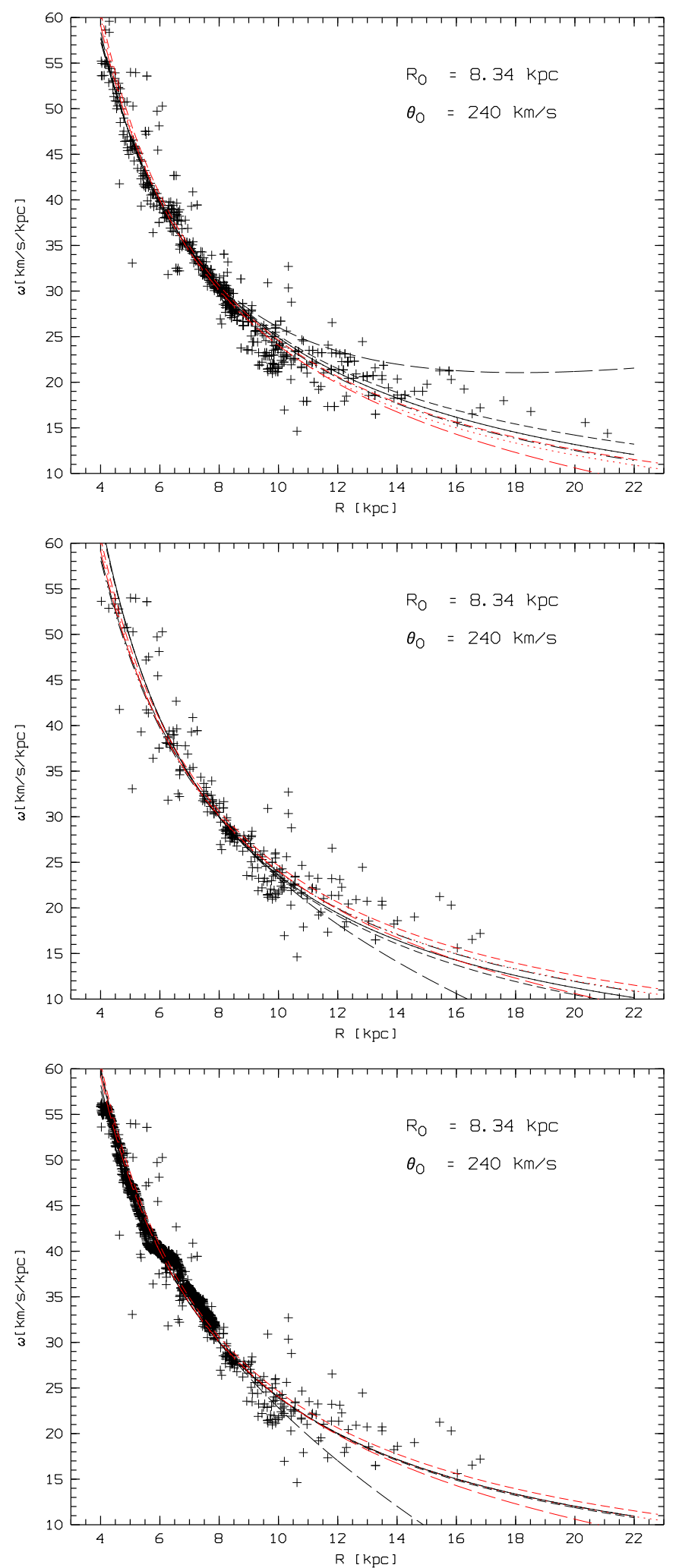

Fig. A.3. As in Fig. A. 2 but for $R_{0}, \theta_{0}$ set to $8.34 \mathrm{kpc}$ and $240 \mathrm{~km} \mathrm{~s}^{-1}$ respectively. The Reid et al. (2014) polynomial (long dashes), power-law (dotted line) and universal (short dashes) rotation curves are superimposed (in red). 
Table A.1. Fitting results.

\begin{tabular}{|c|c|c|c|}
\hline & Sub 123 & Sub 12 & Sub 124 \\
\hline $\begin{array}{l}8.5 / 220 \\
\text { Poly. }\end{array}$ & $\begin{array}{l}a_{1}=1.025 \pm 4 \times 10^{-6} \\
a_{2}=-0.013 \pm 6 \times 10^{-5} \\
a_{3}=-0.147 \pm 1 \times 10^{-4} \\
\chi^{2}=0.213\end{array}$ & $\begin{array}{l}a_{1}=0.996 \pm 6 \times 10^{-4} \\
a_{2}=-0.204 \pm 0.011 \\
a_{3}=-0.198 \pm 0.043 \\
\chi^{2}=0.422\end{array}$ & $\begin{array}{l}a_{1}=0.998 \pm 5 \times 10^{-4} \\
a_{2}=-0.259 \pm 8 \times 10^{-3} \\
a_{3}=-0.477 \pm 2 \times 10^{-2} \\
\chi^{2}=0.186\end{array}$ \\
\hline $\begin{array}{l}8.5 / 220 \\
\text { Power law }\end{array}$ & $\begin{array}{l}a_{1}=1.027 \pm 3 \times 10^{-6} \\
a_{2}=0.0352 \pm 2 \times 10^{-5} \\
\chi^{2}=0.175\end{array}$ & $\begin{array}{l}a_{1}=0.995 \pm 6 \times 10^{-4} \\
a_{2}=-0.179 \pm 0.011 \\
\chi^{2}=0.289\end{array}$ & $\begin{array}{l}a_{1}=1.000 \pm 5 \times 10^{-4} \\
a_{2}=-0.062 \pm 4 \times 10^{-3} \\
\chi^{2}=0.099\end{array}$ \\
\hline $\begin{array}{l}8.5 / 220 . \\
\text { BB } \\
\text { Power law }\end{array}$ & $\begin{array}{l}a_{1}=1.156 \pm 1 \times 10^{-3} \\
a_{2}=0.031 \pm 3 \times 10^{-5} \\
a_{3}=-0.128 \pm 1 \times 10^{-3} \\
\chi^{2}=0.185\end{array}$ & $\begin{array}{l}a_{1}=1.342 \pm 0.078 \\
a_{2}=-0.134 \pm 0.010 \\
a_{3}=-0.346 \pm 0.052 \\
\chi^{2}=0.288\end{array}$ & $\begin{array}{l}a_{1}=1.091 \pm 3 \times 10^{-2} \\
a_{2}=-0.057 \pm 5 \times 10^{-3} \\
a_{3}=-0.09 \pm 8 \times 10^{-2} \\
\chi^{2}=0.099\end{array}$ \\
\hline $\begin{array}{l}8.5 / 220 . \\
\text { Univ. law }\end{array}$ & $\begin{array}{l}a_{1}=1.028 \pm 7 \times 10^{-5} \\
a_{2}=0.044 \pm 7 \times 10^{-5} \\
a_{3}=8.5 \pm 0.013 \\
\chi^{2}=0.175\end{array}$ & $\begin{array}{l}a_{1}=0.995 \pm 0.033 \\
a_{2}=-0.206 \pm 0.040 \\
a_{3}=8.49 \pm 1.36 \\
\chi^{2}=0.316\end{array}$ & $\begin{array}{l}a_{1}=0.999 \pm 2 \times 10^{-2} \\
a_{2}=-0.095 \pm 2 \times 10^{-2} \\
a_{3}=8.49 \pm 1.68 \\
\chi^{2}=0.099\end{array}$ \\
\hline $\begin{array}{l}8.5 / 220 . \\
\text { Polyex } \\
\text { Univ. law }\end{array}$ & $\begin{array}{l}a_{1}=1.0091 \pm 2 \times 10^{-4} \\
a_{2}=0.0032 \pm 9 \times 10^{-7} \\
\chi^{2}=0.174\end{array}$ & $\begin{array}{l}a_{1}=0.785 \pm 0.203 \\
a_{2}=-0.00015 \pm 6 \times 10^{-5} \\
\chi^{2}=0.292\end{array}$ & $\begin{array}{l}a_{1}=1.053 \pm 0.400 \\
a_{2}=0.0058 \pm 1.5 \times 10^{-3} \\
\chi^{2}=0.075\end{array}$ \\
\hline $\begin{array}{l}8.34 / 240 . \\
\text { Poly }\end{array}$ & $\begin{array}{l}a_{1}=1.032 \pm 2 \times 10^{-5} \\
a_{2}=0.221 \pm 2 \times 10^{-4} \\
a_{3}=0.216 \pm 4 \times 10^{-4} \\
\chi^{2}=0.215\end{array}$ & $\begin{array}{l}a_{1}=0.996 \pm 6 \times 10^{-4} \\
a_{2}=-0.090 \pm 0.011 \\
a_{3}=-0.244 \pm 0.042 \\
\chi^{2}=0.452\end{array}$ & $\begin{array}{l}a_{1}=0.998 \pm 5 \times 10^{-4} \\
a_{2}=-0.149 \pm 8 \times 10^{-3} \\
a_{3}=-0.422 \pm 2 \times 10^{-2} \\
\chi^{2}=0.160\end{array}$ \\
\hline $\begin{array}{l}8.34 / 240 . \\
\text { Power law }\end{array}$ & $\begin{array}{l}a_{1}=\mathbf{1 . 0 2 2} \pm \mathbf{2} \times \mathbf{1 0}^{-5} \\
a_{2}=\mathbf{0 . 0 8 0 3} \pm \mathbf{4} \times \mathbf{1 0}^{-5} \\
\chi^{2}=\mathbf{0 . 1 6 9}\end{array}$ & $\begin{array}{l}a_{1}=0.996 \pm 5 \times 10^{-4} \\
a_{2}=-0.069 \pm 0.010 \\
\chi^{2}=0.303\end{array}$ & $\begin{array}{l}a_{1}=0.999 \pm 5 \times 10^{-4} \\
a_{2}=0.032 \pm 3 \times 10^{-3} \\
\chi^{2}=0.095\end{array}$ \\
\hline $\begin{array}{l}8.34 / 240 . \\
\text { BB } \\
\text { Power law }\end{array}$ & $\begin{array}{l}a_{1}=0.838 \pm 3 \times 10^{-4} \\
a_{2}=0.0989 \pm 5 \times 10^{-5} \\
a_{3}=0.184 \pm 3 \times 10^{-4} \\
\chi^{2}=0.169\end{array}$ & $\begin{array}{l}a_{1}=1.000 \pm 8 \times 10^{-3} \\
a_{2}=-0.069 \pm 0.010 \\
a_{3}=-0.004 \pm 8 \times 10^{-3} \\
\chi^{2}=0.301\end{array}$ & $\begin{array}{l}a_{1}=0.937 \pm 7 \times 10^{-3} \\
a_{2}=0.003 \pm 4 \times 10^{-3} \\
a_{3}=0.063 \pm 7 \times 10^{-3} \\
\chi^{2}=0.095\end{array}$ \\
\hline $\begin{array}{l}8.34 / 240 . \\
\text { Univ. law }\end{array}$ & $\begin{array}{l}a_{1}=1.071 \pm 6 \times 10^{-4} \\
a_{2}=0.148 \pm 5 \times 10^{-4} \\
a_{3}=11.69 \pm 0.04 \\
\chi^{2}=0.167\end{array}$ & $\begin{array}{l}a_{1}=0.992 \pm 0.026 \\
a_{2}=-0.092 \pm 0.030 \\
a_{3}=8.70 \pm 2.5 \\
\chi^{2}=0.313\end{array}$ & $\begin{array}{l}a_{1}=0.999 \pm 4 \times 10^{-3} \\
a_{2}=-0.629 \pm 5 \times 10^{-3} \\
a_{3}=7.73 \pm 4.98 \\
\chi^{2}=0.097\end{array}$ \\
\hline $\begin{array}{l}8.34 / 240 . \\
\text { Polyex } \\
\text { Univ. law }\end{array}$ & $\begin{array}{l}a_{1}=1.35 \pm 2 \times 10^{-4} \\
a_{2}=0.0028 \pm 3 \times 10^{-6} \\
\chi^{2}=0.173\end{array}$ & $\begin{array}{l}a_{1}=1.12 \pm 0.11 \\
a_{2}=-0.00028 \pm 8 \times 10^{-5} \\
\chi^{2}=0.291\end{array}$ & $\begin{array}{l}a_{1}=1.125 \pm 3 \times 10^{-2} \\
a_{2}=1.23 \times 10^{-3} \pm 7 \times 10^{-5} \\
\chi^{2}=0.090\end{array}$ \\
\hline
\end{tabular}

Notes. Values in bold is our final choice. 